Research Paper

\title{
The Efficacy and Late Toxicities of Computed Tomography-based Brachytherapy with Intracavitary and Interstitial Technique in Advanced Cervical Cancer
}

\author{
Yun-Zhi Dang*, Pei Li*, Jian-Ping Li, Fei Bai, Ying Zhang, Yun-Feng Mu, Wei-Wei Li, Li-Chun Wei ${ }^{\bowtie}$, Mei \\ Shi ${ }^{\bowtie}$ \\ Department of Radiation Oncology, Xijing Hospital, The Fourth Military Medical University, 17 Changle Western Road, Xi'an 710032, China \\ * These authors contributed equally. \\ $\triangle$ Corresponding author: Li-Chun Wei and Mei Shi, Department of Radiation Oncology, Xijing Hospital, The Fourth Military Medical, University, 17 Changle \\ Western Road, 710032, China. Tel +86-29-8477 5425; Fax +86-29-8477 5425; Email: weilichun@fmmu.edu.cn (LCW) and mshifmmu@yahoo.com (MS) \\ ( ) Ivyspring International Publisher. This is an open access article distributed under the terms of the Creative Commons Attribution (CC BY-NC) license \\ (https://creativecommons.org/licenses/by-nc/4.0/). See http://ivyspring.com/terms for full terms and conditions.
}

Received: 2017.11.22; Accepted: 2018.01.24; Published: 2018.04.18

\begin{abstract}
Purpose: To report the efficacy and late side effects(LSEs) of CT-based image-guided brachytherapy for the treatment of cervical cancer.

Materials: Between 2008 and 2014, 100 patients with FIGO stage IIB-IVA cervical carcinoma were analyzed. The patients received pelvic irradiation (45-50 Gy in 25 fractions) with concurrent chemotherapy, whereas the mean prescribed EBRT dose, including initial and boost doses to positive lymph nodes, ranged from 54 to 64 Gy. Afterwards, intracavitary(IC) or combined intracavitary/interstitial(IC/IS) brachytherapy was performed using a CT-based procedure with prescribed doses of 6 or 8 Gy in 3-7 fractions.

Results: The median follow-up time was 46 months. The 5-year local control, distant metastasis-free survival, and overall survival rates were $88.9 \%, 81.8 \%, 77.9 \%$, respectively. IC/IS brachytherapy improved the HR-CTV D90 compared with IC $(p<0.01)$. Seven patients $(7.0 \%)$ had grade 2 bladder LSEs and none had grade $3 / 4$ bladder LSEs. There was no significant relationship between bladder LSEs and the dose-volume histogram ( $p>0.05$ for all). Thirty-seven patients (37\%) had grade 2 rectal LSEs, $3(3 \%)$ had grade 3 rectal LSE. The rectum $D_{1 \mathrm{lcc}}, D_{2 c c}$, and $D_{5 c c}$ values were significantly higher in patients with grades $2 / 3$ rectal toxicity than in those with grades $0 / 1 \quad(p<0.05$ for all). There was no grade 2 and above small bowel LSEs.

Conclusions: CT-based brachytherapy planning can achieve excellent local control with acceptable morbidity. HR-CTV D90 can increase in the IC/IS group compared with the IC group. The $D_{1 c c}, D_{2 c c}$, and $D_{5 c c}$ all showed excellent predictive values for rectal LSEs.
\end{abstract}

Key words: cervical cancer, dose-volume histogram, brachytherapy, late side effects, computed tomography

\section{Introduction}

A combination of external beam radiotherapy (EBRT), concurrent cisplatin-based chemotherapy, and brachytherapy is the standard treatment for locally advanced cervical cancer ${ }^{[1]}$. Brachytherapy provides a high dose of radiation to the area of disease while sparing the surrounding normal structures. Accordingly, prospective trials that compared 2D and
3D brachytherapy have shown that the latter method exhibits improvements in both toxicity and local control rates $\left.{ }^{[2,} 3\right]$. The Groupe Européen de Curiethérapie (GEC) and the European Society for Radiotherapy and Oncology (ESTRO) jointly published guidelines for magnetic resonance imaging (MRI)-based planning of intracavitary (IC) 
brachytherapy in cervical cancer. They recommended reporting dose-volume histogram (DVH) parameters for OARs in small volumes such as $\mathrm{D}_{0.1 \mathrm{cc}}$ (the minimal dose evaluated in the most exposed $0.1 \mathrm{cc}$ of the organ) and $\mathrm{D}_{2 \mathrm{cc}}[4,5]$.

It is reported that MRI is more reliable than computed tomography (CT) for demarcating the tumor and tumor-involved parametrium in patients with cervical cancer[6]. However, MRI equipment is either located too remotely or is entirely unavailable to some radiation oncology clinics. The adoption of MR-guided brachytherapy in developing countries still has many difficult in access and expense. Since CT simulators are increasing availability in Radiation oncology departments, CT brachytherapy planning has been showed in many studies to be feasible since 2007[7-9].

CT-based brachytherapy optimizes treatment by enabling physicians to decrease the dose to critical organs such as the bladder and bowel, while it can also prescribe the dose to point A or to the high-risk clinical target volume (HR-CTV) ${ }^{[10]}$. This allows for dose escalation to the tumor while maintaining safe doses to the OARs. Since most of the consensus regarding 3D brachytherapy has been achieved based on MRI, analyze the relationship between brachytherapy DVH parameters and clinical outcomes using CT-based planning should be more explored with sufficient follow-up periods.

In this study, we analyzed the CT-guided brachytherapy in a large cohort of patients with locally advanced cervical cancer to investigate clinical outcomes, toxicities, and dosimetric finding of this method.

\section{Methods}

\section{Patient selection and treatment}

Between January 2008 and December 2014, all primary cervical cancer patients treated with curative intent at our center and received CT-based brachytherapy with new plan for every time were included in this study. All patients on this study had no-metastatic disease and staged according to International Federation of Gynecology and Obstetrics (FIGO) classification. The study was approved by the local Institutional Review Board. Prior to RT, all patients underwent a pelvic examination, chest radiography, and pelvic MRI with/without CT.

\section{Treatment}

The EBRT was performed using 3D conformal radiation and the entire pelvic irradiation dose was 45-50 Gy in 25 fractions with concurrent cisplatin (40 $\mathrm{mg} / \mathrm{m}^{2}$ per week), whereas the mean prescribed
EBRT dose, including initial and boost doses to positive lymph nodes, ranged from 54 to $64 \mathrm{~Gy}$. After patients had completed 36-40 Gy in 18-20 fractions, they underwent MRI to evaluate the therapy's effect unless they were not suitable, in which case CT with contrast was performed instead.

After completing EBRT, IC, interstitial (IS), or combined IC/IS brachytherapy was performed using a CT-based procedure. The prescribed doses were 6 or 8 Gy in 4-7 fractions (doses ranged from $24 \mathrm{~Gy} / 3$ fractions to $42 \mathrm{~Gy} / 6$ fractions) once or twice a week. The brachytherapy applicator was inserted under surface anesthesia. Patients were treated with a high-dose-rate iridium-192 source according to a treatment planning system (Nucletron Systems, Veenendaal, The Netherlands). Proper preparation of the bowel and bladder was conducted before CT-based. On the day before brachytherapy, a soaking solution with folium sennae was ingested to completely empty sigmoid and rectum. On the treatment day, 100-120 mL saline was instilled into the empty bladder before the CT scan and BT implementation. The CT-compatible Nucletron Standard tandem-ovoid applicator was inserted and the vagina was packed with gauze to fix the applicator and to increase the distance between the radiation source and the rectum and bladder. IS needles were used in patients with large tumors and poor responses to EBRT. The pelvic transverse images were acquired using a CT simulator (Philips Medical Systems, Cleveland, USA) and transferred to a treatment planning system. The slice thickness of the CT scan was $3 \mathrm{~mm}$, the upper border was crista iliaca while the lower border was at a level $3 \mathrm{~cm}$ below the ischial tuberosity. Based on the CT-standardized contour report[7], reference MRI image, and Gynecological GEC ESTRO working group recommendations ${ }^{[4,11]}$, the HR-CTV, intermediate-risk clinical target volume (IR-CTV), and OARs (intestine, rectum, sigmoid, and bladder) were contoured via CT. The HR-CTV included the entire cervix and the macroscopic extent of the tumor at brachytherapy plus any residual tissue in the parametria. The IR-CTV encompassed either the tumor extension at diagnosis or a $1 \mathrm{~cm}$ margin around the HR-CTV. The outer walls of the OARs were delineated according to the GEC-ESTRO protocol. The rectum was contoured from the anorectal junction to the rectosigmoid flexure. The sigmoid colon delineation commenced at the rectosigmoid and ended $2 \mathrm{~cm}$ above the uterus (unless the descending colon started below this level, in which case the sigmoid colon was delineated cranially up to the transition into descending colon). Small bowel loops lying in the vicinity of the uterus were delineated. The plan for the HR-CTV was 
calculated by using the treatment planning system and then implemented using the Nucletron system.

The doses delivered to the organs at risk were evaluated using the DVH parameters $\mathrm{D}_{0.1 \mathrm{cc}}, \mathrm{D}_{1 \mathrm{cc}}, \mathrm{D}_{2 \mathrm{cc}}$, and $D_{5 c c}$, representing the minimum dose calculated at the most irradiated $0.1,1,2$, and 5 cc volumes, respectively. All doses reported here are based on the total (EBRT plus brachytherapy) and were converted into the biologically equivalent dose in 2 Gy fractions (EQD2). EQD2 used the linear quadratic model with $\alpha / \beta=10$ Gy for the tumor and $\alpha / \beta=3$ Gy for the OARs.

\section{Follow-up}

Patients were reviewed at 1 and 3 months after treatment for the resolution of acute side effects. Subsequently, they received follow-up at 3-6 month intervals by a radiation oncologist and gynecologist. A pelvic examination, ultrasound, CT or MRI, were performed at every visit. Complete response was determined on pelvic examination and imaging results. If abnormalities were found, biopsies were acquired as appropriate. The treating physician according to Radiation Therapy Oncology Group criteria graded acute or late toxicities and the highest grade was recorded. Any intervention required by the patient owing to toxicity was also recorded.

\section{Statistical analysis}

Statistical analysis was conducted using SPSS 18.0 (SPSS, Chicago, IL, USA). Treatment failures were sorted according to the site(s) of first tumoral relapse and were defined as local (cervix, vagina, parametria), pelvic node and distant metastases. Time intervals for local control rate (LC) and distant metastasis-free survival (DFS) and overall rate (OS) were calculated from the date of completion of radiotherapy to the date of event or the last time follow-up. LC, DFS and OS all were calculated using the Kaplan-Meier method. The measurement data, including $\mathrm{DVH}$ parameters, were compared by analysis of variance (ANOVA) or t-test, whereas categorical data (such as the LSE grade) were compared by using the chi-square test.

\section{Results}

\section{Patient characteristics and clinical results}

Between 2008 and 2014, 100 patients with FIGO stage IIB-IVA cervical carcinoma were analyzed. The patient and tumor characteristics were shown in Table 1. The median follow-up was 46 months (range: 3-107 months). The tumor diameters in 51 patients $(51 \%)$ were larger than $5 \mathrm{~cm}$. The tumors in all patients were of squamous cell carcinoma histology (Table 1).

Table 1. Patient and tumor characteristics $(\mathrm{N}=100)$

\begin{tabular}{ll}
\hline Characteristic & value \\
\hline $\begin{array}{l}\text { Age at primary diagnosis (years) } \\
\text { Tumor diameter (cm) }\end{array}$ & Median: 49 (range: $30-73)$ \\
Tumor stage (FIGO stage $\left.{ }^{*}\right)$ & \\
IIB & $24(24 \%)$ \\
IIIB & $70(70 \%)$ \\
IVA & $6(6 \%)$ \\
Histological type & \\
Squamous carcinoma & $100(100 \%)$ \\
LN status & \\
negative & $45(45 \%)$ \\
positive & $55(55 \%)$ \\
Brachytherapy method & \\
Intracavitary & $48(48 \%)$ \\
Combined intracavitary/interstitial & $52(52 \%)$ \\
Brachytherapy Fractions & \\
$3-5$ & $59(59 \%)$ \\
$6-7$ & $41(41 \%)$ \\
Overall treatment time (days) & \\
$<58$ & $42(42 \%)$ \\
$\geq 58$ & $58(58 \%)$ \\
Weekly cisplatin & $5(5 \%)$ \\
No & $95(95 \%)$ \\
yes & Median: 46 (range: 3-107) \\
Follow-up period (months) & \\
\hline *FIGO: International Federation of Gynecology and Obstetrics \\
\end{tabular}

Pelvic recurrence discovered in 10 patients. Moreover, 17 patients experienced distant metastasis and 22 died (the 5-year LC, DFS and OS were $88.9 \%$, $81.8 \%, 77.9 \%$, respectively) (Figure 1a).

The 5-year LC rates were $100 \%$ for patients with stage IIB, $84.2 \%$ for those with stage IIIB, and $100 \%$ for those with stage IVA disease. The 5-year DMFS rates were $86.3 \%$ for patients with stage IIB, $81.8 \%$ for those with stage IIIB, and $66.7 \%$ for those with stage IVA
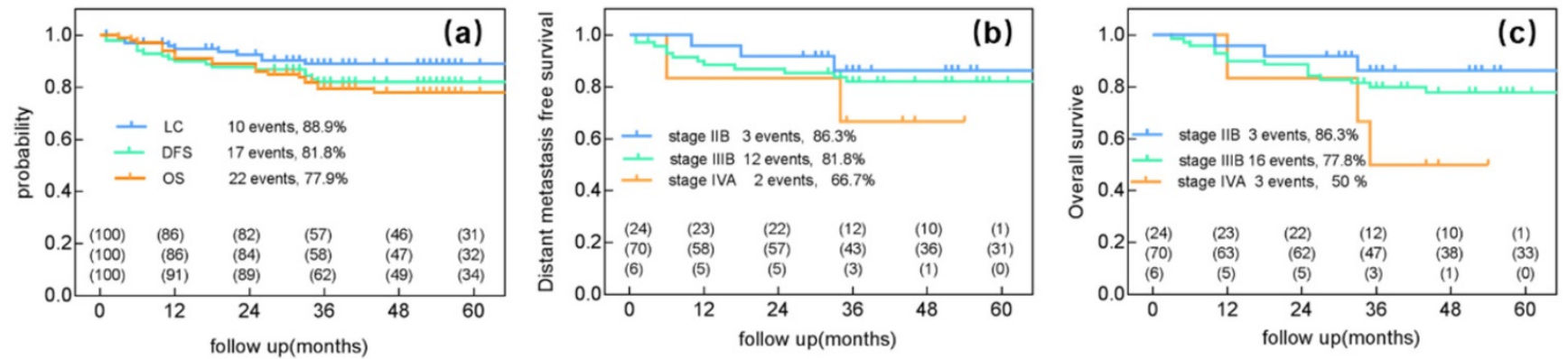

Figure 1. Clinical outcome after radiotherapy \pm chemotherapy and image-guided adaptive brachytherapy. (a) Local control, Distant metastasis-free survival and overall survival for all 100 patients. (b) Distant metastasis-free survival for stage IIB, IIIB, IVA. (c) Overall survival for FIGO stages IIB, IIIB, IVA. 
(Figure $1 \mathrm{~b}$ ). The 5-year OS rates were $86.3 \%$ for patients with stage IIB, $77.8 \%$ for those with stage IIIB, and $50 \%$ for those with stage IVA disease (Figure 1c).

The mean ( \pm standard deviation) $\mathrm{D}_{90}$ values for the HR-CTV and point A were $84.8 \pm 11.6 \mathrm{~Gy}$ and $74.3 \pm 12.9 \mathrm{~Gy}$, respectively.

\section{IC/IS brachytherapy improved the HR-CTV D90 compared with IC}

Combined IC/IS brachytherapy was used in 52\% of the patients. Patient related characteristics of the IC and IC/IS groups were summarized in Table 2 . Patients related factors including the age, FIGO-stage, D2cc for organ at risk (OAR), and treatment time were equally distributed within the two groups. There were no significant differences in $\mathrm{D}_{2 c c}$ for small bowel, bladder and rectum between the two groups $(\mathrm{p}=0.47$, $0.34,0.20$, respectively). Univariate analysis showed no different between bladder and rectum late morbidity of the two groups.

The $\mathrm{D}_{90}$ dose were $89.29 \pm 10.9$ and $79.96 \pm 10.45$ for IC/IS and IC group, respectively $(p<0.01)$. LC in the IC/IS group was $91.6 \%$ at 4 -year compared to $86.3 \%$ at 4-year in the IC group. Though Local control was about $5 \%$ higher in the IC/IS group compared with the IC group, this difference was not significant $(\mathrm{p}=0.45)$.

Table 2. Clinical parameters and treatment outcomes between IC and IC/IS group

\begin{tabular}{llll}
\hline Characteristic & IC group(n=48) & IC/IS group(n=52) & p-value \\
\hline Diameter of the tumor $(\mathbf{c m})$ & & & \\
$\leq 4 \mathrm{~cm}$ & 18 & 19 & 0.9 \\
$>4 \mathrm{~cm}$ & 30 & 33 & \\
Tumor stage (FIGO stage) & & & 0.99 \\
IIB & 16 & 8 & \\
IIIB & 29 & 41 & \\
IVA & 3 & 3 & 0.47 \\
D2cc for OAR & & & 0.34 \\
Small bowel D2cc & $61.23 \pm 7.45$ & $60.14 \pm 7.56$ & 0.20 \\
Bladder D2cc & $72.02 \pm 8.27$ & $73.58 \pm 8.06$ & \\
Rectum D 2cc & $73.55 \pm 6.54$ & $71.84 \pm 6.69$ & 0.25 \\
Bladder LSE & & & \\
Grade 0-1 & 46 & 47 & \\
Grade 2 & 2 & 5 & 0.09 \\
Rectum LSE & & & \\
Grade 0-1 & 25 & 35 & 0.9 \\
Grade 2-3 & 23 & 17 & $<0.01^{*}$ \\
Overall treatment time (days) & & & 0.45 \\
$<58$ & 20 & 30 & \\
$\geq 58$ & 28 & $89.29 \pm 10.9$ & \\
D90 & $79.96 \pm 10.45$ & & \\
LC & $86.3 \%$ & & \\
\hline
\end{tabular}

\section{DVH parameters were predictors of rectum LSEs}

Thirty-seven patients (37\%) were found to have grade 2 rectal LSEs, 3 (3\%) had grade 3, and none had grade 4 . The most common events reported in patients with grade 1 LSE were slight diarrhea and abdominal pain. Patients with grade 2 LSEs experienced similar symptoms as well as intermittent bloody stool. Most patients with grade 2 LSEs were treated with mesalazine. All events reported in patients with grade 3 LSEs were continuous bloody stool requiring transfusion of red cells. None of the patients had fistulas. The mean $\mathrm{D}_{0.1 \mathrm{cc}}, \mathrm{D}_{1 \mathrm{cc}}, \mathrm{D}_{2 \mathrm{cc}}$, and $\mathrm{D}_{5 \mathrm{cc}}$ doses to the rectum in all patients were 83.5 \pm 9.2 , $75.9 \pm 7.3,72.6 \pm 6.6$, and $67.4 \pm 6.2 \mathrm{~Gy}$, respectively. The $\mathrm{D}_{1 \mathrm{cc}}, \mathrm{D}_{2 \mathrm{cc}}$, and $\mathrm{D}_{5 \mathrm{cc}}$ values were significantly higher in patients with grade $2 / 3$ rectal toxicity than in those with grade $0 / 1 \quad(p=0.03, p=0.02$, and 0.02 , respectively), while the $D_{0.1 c c}$ value was not significantly different $(p=0.05)$. The mean $\mathrm{D}_{2 \mathrm{cc}}$ to the rectum in patients with grade $2 / 3$ rectal LSEs was $74.5 \pm 5.6$ Gy. The estimated probability of grade $\geq 2$ rectal LSEs according to the dose-volumetric parameters are shown in Figure 2.

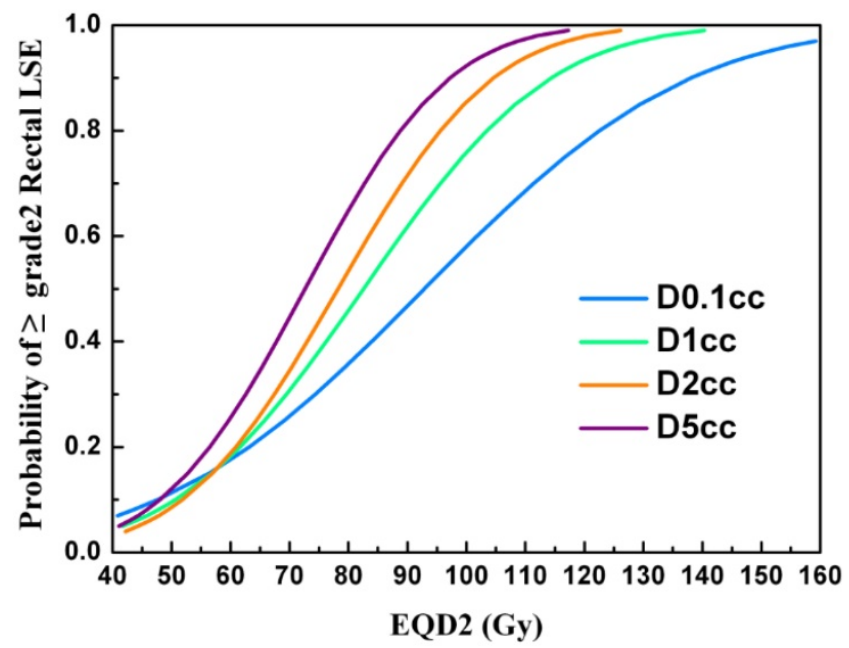

Figure 2. The estimated probability of grade $\geq 2$ rectal LSE according to the dose-volumetric parameters $\left(\mathrm{D}_{0.1 \mathrm{lcc}}, \mathrm{D}_{\mathrm{lcc}}, \mathrm{D}_{2 \mathrm{cc}}\right.$, and $\left.\mathrm{D}_{5 \mathrm{cc}}\right)$.

The patient characteristics that we investigated, including age, tumor diameter, overall treatment time, IC vs IC/IS, and fraction schedule, showed no significant association with the occurrence of rectal LSEs (Table 3). The median interval from the time of completion of radiotherapy to the onset of rectal LSEs was 11 months (range: 1-45 months) and the median last duration was 12 months (range: 1-33 months).

\section{DVH parameters were not predictors of bladder or small bowel LSEs}

Seven patients $(7.0 \%)$ had grade 2 bladder LSEs, while none had grade $3 / 4$ LSEs. The median interval from the time of radiotherapy completion to the onset of bladder LSEs was 12 months (range: 3-17 months). The median last duration was 6.6 months (range: 1-17 months). The mean $\mathrm{D}_{0.1 \mathrm{cc},} \mathrm{D}_{1 \mathrm{cc},} \mathrm{D}_{2 \mathrm{cc}}$, and $\mathrm{D}_{5 \mathrm{cc}}$ values for 
all the patients with bladder LSEs were $83.7 \pm 10.2$, $76.1 \pm 8.7,72.8 \pm 8.1$, and $66.7 \pm 6.9 \mathrm{~Gy}$, respectively. There was no significant relationship between LSEs and the DVH ( $p=0.307,0.248,0.227$, and 0.181 , respectively).

Table 3. Relationship between rectal late side effects and clinical parameters

\begin{tabular}{llll}
\hline Characteristic & Grade $\mathbf{0 - 1}$ & Grade 2-3 & p-value \\
\hline Age & 38 & 22 & \\
$<50$ & 20 & 20 & 0.218 \\
$\geq 50$ & & & \\
Diameter of the tumor (cm) & 19 & 18 & 0.17 \\
$\leq 4 \mathrm{~cm}$ & 41 & 22 & \\
$>4 \mathrm{~cm}$ & & & \\
Brachytherapy method & 25 & 23 & 0.12 \\
Intracavitary & 35 & 17 & \\
Combined intracavitary/interstitial & & & \\
Fractions & 49 & 10 & 1.0 \\
$3-5$ & 34 & 7 & \\
$6-7$ & & & \\
Overall treatment time (days) & 22 & 20 & \\
$<58$ & 38 & 20 & \\
$\geq 58$ & &
\end{tabular}

In our study, we limited the dose to the small bowel to below $64 \mathrm{~Gy}$ in all patients. The mean $\mathrm{D}_{0.1 \mathrm{cc}}$ $\mathrm{D}_{1 \mathrm{cc}}, \mathrm{D}_{2 \mathrm{cc}}$, and $\mathrm{D}_{5 \mathrm{cc}}$ values to the small bowel were $67.4 \pm 11.5,62.44 \pm 8.4,60.66 \pm 7.5$, and $57.3 \pm 5.5 \mathrm{~Gy}$, respectively. At baseline, none of the patients reported chronic small bowel morbidity. Moreover, 80 patients $(80 \%)$ reported no small bowel morbidity at any time during the follow-up period (grade 0), while 20 patients $(20 \%)$ had grade 1 LSEs. The most common events reported were slight diarrhea and discontinuous abdominal pain.

\section{Discussion}

$\begin{array}{rrr}\text { Several } & \text { studies have compared dose } \\ \text { distributions } & \text { between } & \text { conventional }\end{array}$ radiography-based planning and $3 \mathrm{D}$ treatment planning using CT or MRI. All have shown that 3D optimizations can improve tumor target dose coverage while maintaining a safe dose to the OARs[11-17]. Although MRI provides a better delineation of the tumor and involved parametria[6], it is not readily available to many institutions. CT is the most commonly utilized imaging modality for 3D planning of brachytherapy in developing countries. In a study which compares the consensus of CT contours based CT and MRI based brachytherapy, it shows the large volume on CT for all 3 case and there is no significant different in $\mathrm{D}_{2 c c}$ OAR or $\mathrm{D}_{90}$ between $\mathrm{CT}$ and MRI ${ }^{[8]}$. Our study showed with long follow up excellent local control and OS for CT-guided brachytherapy planning using the GEC-ESTRO guidelines for delineation. Moreover, late toxicity was low following adequate bowel and bladder preparation as well as proper plan optimization.

Our study showed excellent clinical outcomes in terms of 5-year LC, DMFS, and OS. The clinical local control rates comparable to those in previous studies of CT or MRI-based brachytherapy. Wang et al.[18] also showed excellent clinical outcomes following CT-based brachytherapy planning for cervical cancer patients. With median follow-up of 15 months, 2 of 20 patients experienced local failure and 1 showed internal iliac nodal metastasis. A recent study by Agnieszka et al.[19] investigated 216 cervical cancer patients who underwent CT-based brachytherapy. The 5-year OS and disease-free survival rates were $66.4 \%$ and 58.5\%, respectively. In 2007, Pötter et al. [20] reported 145 Austrian patients treated with MRI-based image-guided high-dose-rate brachytherapy using a tandem-ring applicator, where they achieved a local control rate of $88 \%$ for the true pelvis at 3 years, as well as an OS rate of $68 \%$. Ribeiro et al.[21] investigated 170 patients who underwent MRI or CT-based brachytherapy, and found a 5-year OS rate of $65 \%$ and a local control rate of $81 \%$.

In our study, only 10 patients experienced local recurrence, this may be because $52 \%$ of our patients underwent IC/IS treatment. The introduction of IC/IS applicators has made possible dose distributions with higher conformity, especially for eccentric cervical tumors[22]. In our study, we implemented IC/IS treatment for patients with persistent or eccentric tumors. The mean $\mathrm{D}_{90}$ was significantly higher in the IC/IS group compared to the IC-only group. Though Local control was about $5 \%$ higher in the IC/IS group compared with the IC group, the difference was not significant $(p=0.52)$. However, this difference was not significant $(p=0.52)$, this maybe because the small size of patients. Additionally, there were no increases in grade $2 / 3$ rectal LSEs when IS needles were used. These findings were consistent with previous study which found combined IC/IS brachytherapy enabled tumor-specific dose escalation and resulted in significantly better local control in large tumors, without increased treatment-related late morbidities[23]. For the recurrent cervical cancer, cisplatin and paclitaxel may be the acceptable chemotherapy regimens ${ }^{[24]}$.

In our study, $37 \%$ and $3 \%$ of the patients experienced grade 2 and grade 3 rectal LSEs, respectively. There were no grade 4 LSEs. Additionally, the $\mathrm{D}_{0.1 \mathrm{cc}}, \mathrm{D}_{1 \mathrm{cc}}, \mathrm{D}_{2 \mathrm{cc}}$, and $\mathrm{D}_{5 \mathrm{cc}}$ doses to the rectum were shown to be predictive of LSEs, as was reported in a previous study. In our investigation, the $D_{2 c c}$ to the rectum in most patients was limited to 75 Gy, which was in accordance with the recommendation of the Gynecological GEC-ESTRO 
working group. The mean rectal $\mathrm{D}_{2 c c}$ was $72.6 \pm 6.6 \mathrm{~Gy}$. The rectal toxicity rates were comparable to those in previous CT or MRI-guided brachytherapy studies (Table 4) ${ }^{[9,19,25,26]}$. However, Beant et al[ ${ }^{[27]}$ reported a gastrointestinal or genitourinary LSE rate of only $0.9 \%$ for grade 3 or above at 2 years. This may be because the use of intensity-modulated radiotherapy in their series decreased the incidence of long-term toxicity. Our study also suggested that the $\mathrm{D}_{5 \mathrm{cc}}$ may be another powerful factor in predicting rectal LSEs, which was consistent to previous finding ${ }^{[28]}$.

Table 4. Comparison of late toxicity rates among studies using computed tomography or magnetic resonance imaging planning for brachytherapy

\begin{tabular}{llllll}
\hline Study & $\begin{array}{l}\text { Patients } \\
\text { (n) }\end{array}$ & $\begin{array}{l}\text { Follow-up CT or MRI } \\
\text { (years) }\end{array}$ & $\begin{array}{l}\text { Grade 3 } \\
\text { bladder } \\
\text { toxicity (\%) }\end{array}$ & $\begin{array}{l}\text { Grade 3 } \\
\text { rectal } \\
\text { toxicity (\%) }\end{array}$ \\
\hline Tan et al.[25] & 28 & 3 & MRI & 0 & 11 \\
Pötter et al.[26] & 189 & 3 & MRI & 2.9 & 6.1 \\
$\begin{array}{l}\text { Vicky et al.[9] } \\
\text { Ribeiro et al.[21] }\end{array}$ & 95 & 3 & MRI & 2 & 11 \\
$\begin{array}{l}\text { Agnieszka et } \\
\text { al.[19] }\end{array}$ & 216 & 3 & MRI or CT & 6 & 5 \\
Present study & 100 & 4 & CT & 3.3 & 6 \\
\hline
\end{tabular}

In our study, we limited all the bladder $\mathrm{D}_{2 c c}$ values to under 78 Gy since the bladder has a higher tolerance to radiation, and the mean $\mathrm{D}_{2 \mathrm{cc}}$ to the bladder was $72.8 \pm 8.1 \mathrm{~Gy}$. Only 7 patients experienced grade 2 bladder LSEs, while none experienced grade 3 or above LSEs. Petra et al.[29] investigated 141 cervical cancer patients for bladder side effects, and observed a significant dose effect for all DVH parameters as related to grade 2 complications. We found no significant relationship between bladder LSE and $\mathrm{DVH}$, this may have been attributable to the lower incidence of LSE grades $\geq 2$ in our study.

To date, there are few reports on dose-volume effect relationships for the small bowel. Petit et al.[30] found that small bowel $\mathrm{D}_{0.1 \mathrm{cc}}$, and $\mathrm{D}_{2 \mathrm{cc}}$ were not predictive of late small bowel morbidity. In our study, the mean $\mathrm{D}_{2 \mathrm{cc}}$ to the small bowel was $60.66 \pm 7.5 \mathrm{~Gy}$, there were no grade 2 and above LSEs. However, because we limited the dose to the small bowel to $<64$ Gy in all patients, the highest-tolerated dose for the small bowel is still unclear. Furthermore, the dose-effect relationships are yet to be established for the sigmoid[31].

The advantage of this study was the large cohort size as well as the long follow-up times that produced mature results. To our knowledge, there are relatively few studies that have investigated the relationship between DVH parameters and efficacy, as well as late toxicities to OARs, using CT-based brachytherapy. A limitation of this study, however, is its retrospective nature.

\section{Conclusion}

CT-based brachytherapy for the treatment of locally advanced cervical cancer results in high local control rates and excellent OS. The DVH parameters can predict the incidence and grades of LSEs. There was no grade 3 bladder LSEs when the mean $D_{2 c c}$ was 72.8 Gy. The mean rectal $\mathrm{D}_{2 c c}$ in our patients with grade $2 / 3$ rectal LSE was $74.5 \pm 5.6 \mathrm{~Gy}$. Moreover, a mean $\mathrm{D}_{2 \mathrm{cc}}$ of $60.6 \mathrm{~Gy}$ to the small bowel was deemed safe.

\section{Acknowledgements}

This work is supported by the grants from National Natural Science Foundation of China (No. 81272346).

\section{Competing Interests}

The authors have declared that no competing interest exists.

\section{References}

1. Tanderup, K., P.J. Eifel, C.M. Yashar, et al. Curative radiation therapy for locally advanced cervical cancer: brachytherapy is not optional. Int J Radiat Oncol Biol Phys. 2014; 88(3): 537-539.

2. Charra-Brunaud, C., V. Harter, M. Delannes, et al. Impact of 3D image-based PDR brachytherapy on outcome of patients treated for cervix carcinoma in France: Results of the French STIC prospective study. Radiother Oncol. 2012; 103(3): 305-313.

3. Kang, H.-C., K.H. Shin, S.-Y. Park, et al. 3D CT-based high-dose-rate brachytherapy for cervical cancer: Clinical impact on late rectal bleeding and local control. Radiother Oncol. 2010; 97(3): 507-513.

4. Haie-Meder, C., R. Pötter, E. Van Limbergen, et al. Recommendations from Gynaecological (GYN) GEC-ESTRO Working Group (I): concepts and terms in 3D image-based 3D treatment planning in cervix cancer brachytherapy with emphasis on MRI assessment of GTV and CTV. Radiother Oncol. 2005; 74(3): 235-245.

5. Pötter, R., C. Haie-Meder, E.V. Limbergen, et al. Recommendations from gynaecological (GYN) GEC ESTRO working group (II): Concepts and terms in 3D image-based treatment planning in cervix cancer brachytherapy-3D dose volume parameters and aspects of $3 \mathrm{D}$ image-based anatomy, radiation physics, radiobiology. Radiother Oncol. 2006; 78(1): 67-77.

6. Hricak, H., C. Gatsonis, F.V. Coakley, et al. Early invasive cervical cancer: CT and MR imaging in preoperative evaluation-ACRIN/GOG comparative study of diagnostic performance and interobserver variability. Radiology. 2007; 245(2): 491-498.

7. Viswanathan, A.N., J. Dimopoulos, C. Kirisits, et al., Computed tomography versus magnetic resonance imaging-based contouring in cervical cancer brachytherapy: results of a prospective trial and preliminary guidelines for standardized contours. Int J Radiat Oncol Biol Phys. 2007; 68(2): 491-498.

8. Viswanathan, A.N., B. Erickson, D.K. Gaffney, et al. Comparison and consensus guidelines for delineation of clinical target volume for CT- and MR-based brachytherapy in locally advanced cervical cancer. Int J Radiat Oncol Biol Phys. 2014; 90(2): 320-328.

9. Koh, V., B.A. Choo, K.M. Lee, et al. Feasibility study of toxicity outcomes using GEC-ESTRO contouring guidelines on CT based instead of MRI-based planning in locally advanced cervical cancer patients. Brachytherapy. 2017; 16(1): 126-132.

10. Wanderas, A.D., M. Sundset, I. Langdal, et al. Adaptive brachytherapy of cervical cancer, comparison of conventional point A and CT based individual treatment planning. Acta Oncol. 2012; 51(3): 345-354.

11. Hellebust, T.P., C. Kirisits, D. Berger, et al. Recommendations from gynaecological (GYN) GEC-ESTRO working group: considerations and pitfalls in commissioning and applicator reconstruction in 3D image-based treatment planning of cervix cancer brachytherapy. Radiother Oncol. 2010; 96(2): 153-160.

12. Dimopoulos, J.C.A., C. Kirisits, P. Petric, et al. The vienna applicator for combined intracavitary and interstitial brachytherapy of cervical cancer: Clinical feasibility and preliminary results. Int J Radiat Oncol Biol Phys. 2006; 66(1): 83-90.

13. Lindegaard, J.C., K. Tanderup, S.K. Nielsen, et al. MRI-guided 3D optimization significantly improves DVH parameters of pulsed-dose-rate 
brachytherapy in locally advanced cervical cancer. Int J Radiat Oncol Biol Phys. 2008. 71(3): 756-764.

14. De Brabandere M, Mousa AG, Nulens A, et al. Potential of dose optimisation in MRI-based PDR brachytherapy of cervix carcinoma. Radiother Oncol. 2008; 88(2): $217-226$

15. Jürgenliemk-Schulz, I.M., R.J.H.A. Tersteeg, J.M. Roesink, et al., MRI-guided treatment-planning optimisation in intracavitary or combined intracavitary/ interstitial PDR brachytherapy using tandem ovoid applicators in locally advanced cervical cancer. Radiother Oncol. 2009; 93(2): 322-330.

16. Tanderup, K., S.K. Nielsen, G.-B. Nyvang, et al. From point A to the sculpted pear: MR image guidance significantly improves tumour dose and sparing of organs at risk in brachytherapy of cervical cancer. Radiother Oncol. 2010; 94(2): $173-180$.

17. Wachter-Gerstner, N., S. Wachter, E. Reinstadler, et al. The impact of sectional imaging on dose escalation in endocavitary HDR-brachytherapy of cervical cancer: results of a prospective comparative trial. Radiother Oncol. 2003; 68(1): 51-59.

18. Wang, Y., W.J. Ye, L.H. Du, et al., Dose-volume parameters and clinical outcome of CT-guided free-hand high-dose-rate interstitial brachytherapy for cervical cancer. Chin J Cancer, 2012; 31(12): 598-604.

19. Zolciak-Siwinska, A, E. Gruszczynska, M. Bijok, et al. Computed tomography-planned high-dose-rate brachytherapy for treating uterine cervical cancer. Int J Radiat Oncol Biol Phys. 2016; 96(1): 87-92.

20. Pötter, R. J. Dimopoulos, P. Georg, et al. Clinical impact of MRI assisted dose volume adaptation and dose escalation in brachytherapy of locally advanced cervix cancer. Radiother Oncol. 2007; 83(2): 148-155.

21. Ribeiro, I., H. Janssen, M. De Brabandere, et al. Long term experience with 3D image guided brachytherapy and clinical outcome in cervical cancer patients. Radiother Oncol. 2016; 120(3): 447-454.

22. Fokdal, L., K. Tanderup, S.B. Hokland, et al. Clinical feasibility of combined intracavitary/interstitial brachytherapy in locally advanced cervical cancer employing MRI with a tandem/ring applicator in situ and virtual preplanning of the interstitial component. Radiother Oncol. 2013; 107(1): 63-68.

23. Fokdal, L., A. Sturdza, R. Mazeron, et al. Image guided adaptive brachytherapy with combined intracavitary and interstitial technique improves the therapeutic ratio in locally advanced cervical cancer: Analysis from the retroEMBRACE study. Radiother Oncol. 2016; 120(3): 434-440.

24. Geisler, J., J. Swathirajan, K. Wood, et al. Treatment of advanced or recurrent cervical cancer with Cisplatin or Cisplatin containing regimens: a cost effective analysis. J Cancer. 2012; 3: 454-458.

25. Tan, L.T. C.E. Coles, C. Hart, et al. Clinical impact of computed tomography-based image-guided brachytherapy for cervix cancer using the tandem-ring applicator-the addenbrooke's experience. Clinical Oncology. 2009; 21(3): 175-182.

26. Pötter, R. T.H. Knocke, C. Fellner, et al. Definitive radiotherapy based on HDR brachytherapy with iridium 192 in uterine cervix carcinoma: report on the vienna university hospital findings (1993-1997) compared to the preceding period in the context of ICRU 38 recommendations. Cancer Radiother. 2000; 4(2): 159-172.

27. Gill, B.S., H. Kim, C.J. Houser, et al. MRI-guided high-dose-rate intracavitary brachytherapy for treatment of cervical cancer: the university of pittsburgh experience. Int J Radiat Oncol Biol Phys. 2015; 91(3): 540-547.

28. Kim, T.H., J.-Y. Kim, D.K. Sohn, et al. A prospective observational study with dose volume parameters predicting rectosigmoidoscopic findings and late rectosigmoid bleeding in patients with uterine cervical cancer treated by definitive radiotherapy. Radiat Oncol, 2013; 8(1): 28-37.

29. Georg, P., R. Pötter, D. Georg, et al. Dose effect relationship for late side effects of the rectum and urinary bladder in magnetic resonance image-guided adaptive cervix cancer brachytherapy. Int J Radiat Oncol Biol Phys. 2012; 82(2): 653-657.

30. Petit, C., I. Dumas, C. Chargari, et al. MRI-guided brachytherapy in locally advanced cervical cancer: Small bowel D0.1 $\mathrm{cm} 3$ and D2 $\mathrm{cm} 3$ are not predictive of late morbidity. Brachytherapy, 2016; 15(4): 463-470.

31. Koom, W.S., D.K. Sohn, J.Y. Kim, et al. Computed tomography-based high-dose-rate intracavitary brachytherapy for uterine cervical cancer: preliminary demonstration of correlation between dose-volume parameters and rectal mucosal changes observed by flexible sigmoidoscopy. Int J Radiat Oncol Biol Phys. 2007; 68(5): 1446-1454. 